\title{
Open Networks: Generalized Multi-Sensor Characterization
}

\author{
Alan N. Steinberg \\ CUBRC, Inc. \\ Bethesda, MD, USA \\ Alan.steinberg@comcast.net
}

\begin{abstract}
This paper examines issues in characterizing the performance of information sources as necessary for data fusion and coordination in a netcentric environment. In many practical applications, interacting agents have various degrees - and possibly time-varying degrees - of allegiance, common purpose, cooperativeness, information fidelity, controllability, etc. Agents share information with friends, foes and innocent bystanders alike, with varying degrees of cooperativeness and openness. In such cases, each network node needs to explicitly estimate the performance, trustworthiness and allegiance of all other contributing nodes as a part of the general multisensor/multi-target state estimation process. A sensor's or information system's reporting bias - which may include intentional or unintentional human biases - is distinguished from its measurement bias. The problem is compared with that of measurement bias estimation, e.g. in target tracking. Formulations for estimation of biases in discrete variable reporting - e.g. in target classification or activity state reporting - are explored.
\end{abstract}

Keywords: reporting bias, situation assessment, threat assessment, closed-loop data fusion, network-centric warfare, network-centric operations, sensor/source characterization, data alignment, registration, calibration, information warfare

\section{Sensor/Source Characterization}

The rôle of many data fusion problems is in estimating and predicting the state of specific types of entities in the external environment: targets, threats, military formations, etc. Ultimately, however, such problems are inseparable from problems of characterizing sensor alignment and performance, and of validating one's library of target and environment models. This has been a fundamental problem in the integration of "legacy" or external sensors into a multisensor data fusion or collection management system. The problem is accentuated in distributed data fusion implementations, as envisioned for Network-Centric Operations. In such implementations virtually all information sources are in effect external systems to any particular fusion-capable platform.

Currently, sensor characterization - to include calibration, alignment and geo-registration - is generally performed as a process separate from the problem of estimating the "external" environment. Sensor noise statistics and biases can be characterized in calibration and registration; either by using (a) internal calibration signal sources, (b) known "fiducial" targets in the environment, or (c) the ensemble of information available in a multi-sensor system.

A more powerful realization of the role of data fusion - and, indeed, of resource management, as well - is one in which all sources are exploited to solve all required state estimation/prediction problems, to include sensor/source characterization as well as external target and situation state estimation.

The estimation and prediction of states of targets, other external entities (threats, terrain, weather, etc.) as well of one's own platform, its sensors and other systems - is a single problem, amenable to a unified self-consistent solution. The evaluation of the system's models of the characteristics and behavior of all of these external and organic entities is likewise a component of the single problem of estimating the actual world state.

The problem of characterizing information sources is present even in systems that have been designed and integrated as a unit.

The problem is made even more difficult when the performance of information sources cannot be assumed. This is the case in network-centric operations, in which calibration and registration are not easily performed.

It is even more the case when the cooperation or common purpose of information sources cannot be assured; e.g. when sources are willful agents with varying degrees of autonomy; raising the possibility of private agendas. The same factors can affect the estimation and controllability of assets having some degree of autonomy. Cases extend to the use of noncooperating agents; e.g. enemy radars that "report" to our ELINT systems who and where they are; generally with no attempt to deceive.

Other cases include purposeful deception, to include such Information Warfare techniques as decoys, deceptive jamming and propaganda. An example of this type of deceptive source is the computer "zombie"; i.e. a computer that has been infected by malicious code that allows spammers to use it to send e-mail [1].

Finally, there are third-party agents - e.g. commercial news sources, reference texts, and the like - that may or may not be unbiased.

Table 1 shows the range of cases in which performance (including data reporting) is systematically biased. $^{1}$

\footnotetext{
${ }^{1}$ In some ways, the idea relates to H. P. Grice's concept of "conversational implicature"; which lays out various presumptions implicit in conversations [2]. The foremost of these presumptions is that people's utterances are expected to serve the purpose of the conversation. In many cases, this
} 
Table 1. Types of systematic biases in information reporting or control response

\begin{tabular}{|c|c|c|}
\hline $\begin{array}{l}\text { Cases of Biased Information } \\
\text { or Biased Response }\end{array}$ & Examples & $\begin{array}{l}\text { Trust- } \\
\text { worthiness }\end{array}$ \\
\hline $\begin{array}{l}\text { FULLY COOPERATIVE } \\
\text { AUTONOMOUS AGENTS } \\
\text { (unintentional reporting \& } \\
\text { response errors) }\end{array}$ & $\begin{array}{l}\text { Integrated, fully } \\
\text { controlled system-of- } \\
\text { systems }\end{array}$ & Low High \\
\hline $\begin{array}{l}\text { SEMI-COOPERATIVE } \\
\text { AGENTS (possibly intentional } \\
\text { reporting \& response errors) }\end{array}$ & Employees, Partners & \\
\hline $\begin{array}{l}\text { DECEPTIVE HOSTILE } \\
\text { AGENTS (likely biased } \\
\text { reporting \& response) }\end{array}$ & $\begin{array}{l}\text { Decoys, false-target } \\
\text { jamming, Information } \\
\text { operations, Computer } \\
\text { viruses, Trojan horses }\end{array}$ & \\
\hline $\begin{array}{l}\text { NON-DECEPTIVE HOSTILE } \\
\text { INFORMATION SOURCES } \\
\text { (only unintentional reporting \& } \\
\text { response errors) }\end{array}$ & $\begin{array}{l}\text { Enemy radars, } \\
\text { Combatant platforms }\end{array}$ & \\
\hline $\begin{array}{l}\text { NON-COOPERATIVE THIRD- } \\
\text { PARTIES SOURCES (possibly } \\
\text { biased reporting \& responses) }\end{array}$ & $\begin{array}{l}\text { Commercial news } \\
\text { sources, Websites }\end{array}$ & \\
\hline
\end{tabular}

Given the general lack of useful calibration sources or fiducial targets in such cases, the data fusion system must characterize the reliability and performance of each source using the ensemble of information. To extend the network-centric concept, this implies that we can't assume clear system boundaries: "us" vs "them": Allegiance is a matter of estimation.

\section{Open Network Concept}

There is value, then, in thinking of the information network as unbounded, with node agents having various degrees - and possibly time-varying degrees - of allegiance, common purpose, cooperativeness, information fidelity, controllability, etc. As illustrated in Figure 1, all agents share information with friends, foes and innocent bystanders alike, with varying degrees of cooperativeness and openness.

The job of every other agent, then, is (in the words of Ronald Reagan when faced with the issue in the 1980s) to "trust but verify"; i.e. to characterize all sources "ours" and "theirs" alike - with a grain of skepticism. Ultimately it is a problem of multisensor/multi-target state estimation, in which sensors themselves are included among the "targets" of estimation. As with calibration of multiple "organic" sources, the ensemble of information is used to calibrate each agent's informational and physical performance.

implies providing truthful and relevant information to one's interlocutors. Some contributions to conversations, however, may have other purposes.

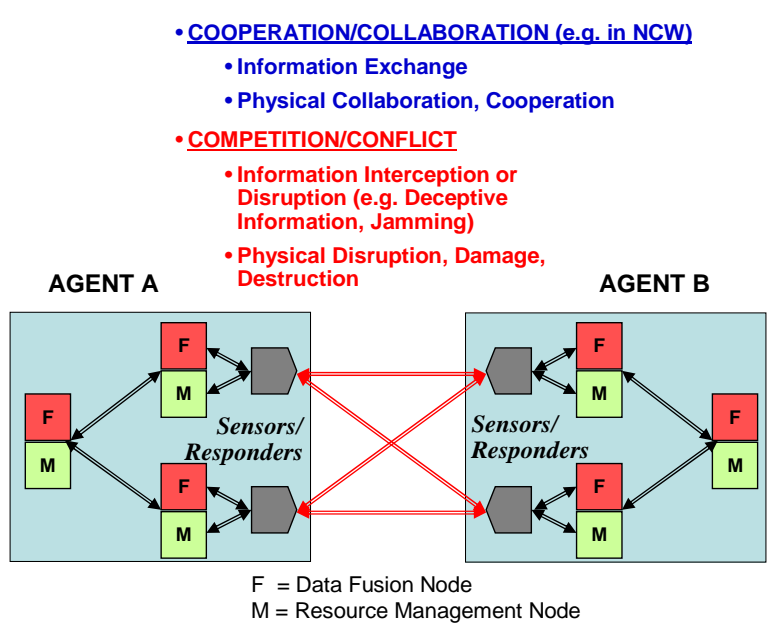

Figure 1. Open-network concept

This concept extends the notion of a Service-Oriented Architecture to include cases of partial and conditional cooperation. It extends the notion of Network-Centric Operations to include partially and conditionally participating agents; and, indeed, to non-cooperative information sources. It extends the conventional Network Centric Warfare notion of "power to the edge" [3] to include networks without edges. ${ }^{2}$

\section{Epistemic and Ontologic Uncertainty}

The problem in many military, intelligence or commercial applications is that the information exploitation system must be adaptive to unanticipated missions, interacting agents and tactics.

For example, in modern anti-terrorism situations, we can't assume knowledge of who will be our adversary, or perhaps that we even have an adversary. We can't assume that adversary's choice of specific objectives, tactics, or weapons. However, we can assume

- An increasing diversity of threat tactics, involving combinations of conventional military forces, unconventional use of force, political and economic tactics and psychological tactics;

- An increasing exploitation of Information Technology, to included sophisticated Communications and coordination, tailored countermeasures, and coordinated multi-faceted attack: military, economic, psychological; and

- An increasing diversity of goals: military, diplomatic, economic, personal, societal, etc.

The approach builds on our recent work in developing systems for Situation and Threat Assessment [4,5]. That work involves developing the theoretical and ontological foundations to enable the representation and recognition of relationships and of threat situations. Such applications require the system to exploit a wide range of evidence and a wide range of entity and aggregate behavior models. This is certainly the case in

\footnotetext{
${ }^{2}$ Or, more exactly stated, networks with fuzzy edges; amenable to representation as fuzzy sets of relationships [4].
} 
the many situations of interest that involve estimating and predicting human individual and group behavior.

An architecture for adaptively building and refining situation estimates is shown in Figure 2. The architecture extends one developed for model-based scene understanding and target recognition.

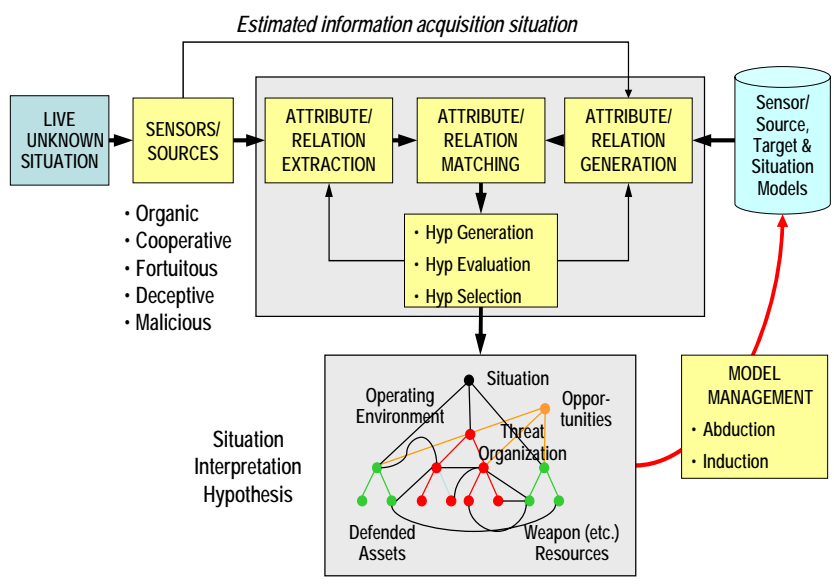

Figure 2. Adaptive situation/threat assessment architecture

Key to the performance of such a system in most practical threat assessment applications is the integration of such processes with processes for model evaluation and model refinement.

Models and methods of threat assessment have been developed in programs for diverse national security and commercial applications $[5,6]$. In general, threats are characterized, predicted and recognized in terms of the indications and constraints on their actions imposed by their capability, opportunity and intent to carry out various actions.

Indeed, this is a general model for characterizing, predicting and recognizing any intentional behavior. Accordingly, the data structure for threat (or predictive behavior) hypotheses that has appeared in previous publications (e.g. [4,5]) has been generalized as shown in Figure 3.

In general, actions can be thought of as involving one or more actors (or "agent") and one or more objects acted upon ("targets”).

- Capability may be defined as the availability of resources sufficient to undertake an action of interest.

- Opportunity is the presence of an operating environment in which potential targets of an action are present and are susceptible to being acted upon.

- Intent, of course, is an element of an agent's will to act.

Without penetrating the murky territory of free-will vs. determinism, we can assert that intent is predictable, at least to some extent, as a function of an agent's objectives.

Somewhat more mysterious are the processes by which a person decomposes objectives and assigns utility to them, or generates candidate actions or estimates their costs and outcomes. Nonetheless, it is this dim realm in which intent assessment must operate.

The adaptive evidence accrual process shown in Figure 2 is used to generate, evaluate and select threat hypotheses having this structure. The capability, opportunity and intent of particular agents to carry out various acts are decomposed into mutually consistent sets and evaluated against the available evidence.

The process iteratively builds and validates Interpretation Hypotheses, which attempt to explain the available evidence. A Feature Extraction process searches available data for indicators (i.e. supporting evidence) of situation types of interest (e.g. movements of weapons, forces or other resources related to threat organizations).

To the extent that such situation types are supported, Hypothesis Generation develops one or more hypotheses as to how the evidence supports candidate instantiations of such situations. These Interpretation Hypotheses have the form of labeled directed graphs as illustrated at the bottom of Figure 2.

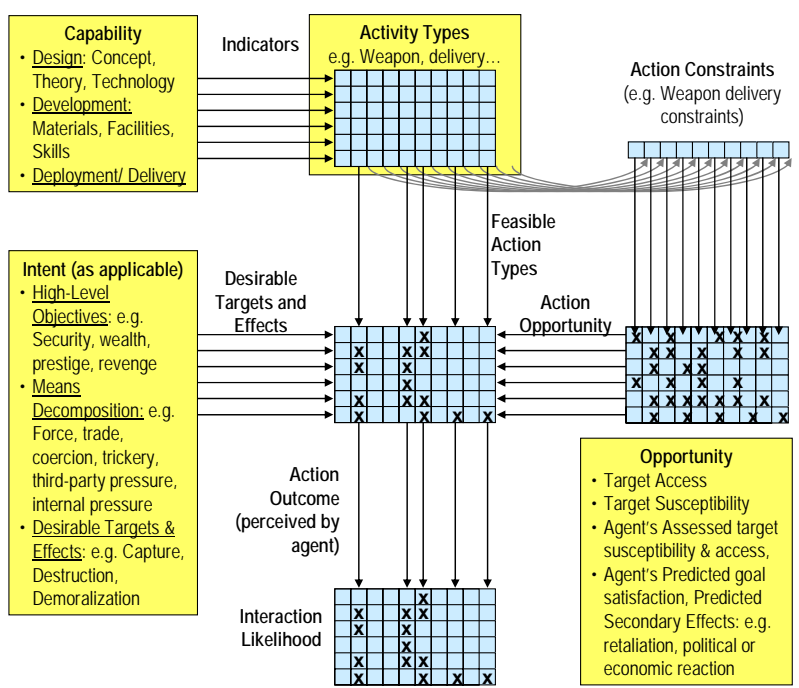

Figure 3. General predictive behavior model in terms of Capability, Opportunity and Intent

Interpretation hypotheses are constructed on the basis of target and situation models, together with sensor/ measurement models.

The latter are used to predict the features expected to be observed given the candidate hypothesis. Features can include directly observable physical features of individuals. They can also include physical relationships among individuals (e.g. spatio-temporal relationships or causal relationships such as shadowing, obscuration, kinetic interaction, etc.).

Hypotheses are evaluated and selected on the basis of their consistency with the evidence; i.e. by the extent to which all the data is explained by the situation hypothesis.

If the evidence is only partially supported, Hypothesis Selection can initiate one or more actions:

- Tasking of Feature Generation to process the model set to identify types of evidence that would either support or refute candidate hypotheses; 
- $\quad$ Tasking of Feature Extraction to process the data to find such evidence in the data (in a closed-loop Information Exploitation system, this can also include tasking of Collection Management);

- $\quad$ Tasking of Hypothesis Generation to refine existing hypotheses to better match the available data.

Note also the integration of model management functions that evaluate and refine the system's models of entity characteristics, relationships and behaviors.

Deduction, abduction and induction all play a part in the above process:

- To the extent that Situation and Threat Assessment involve recognizing situations, they involve recognition processes akin to those found in target recognition. Recognition is paradigmatically a deductive process, in which data are compared via a measurement model with entity states of interest.

- $\quad$ To the extent that Situation and Threat Assessment involve understanding situations, they involve abductive (i.e. explanatory) processes akin to those found in scene understanding.

- To the extent that Situation and Threat Assessment involve predicting situations, they involve inductive, processes akin to those found in target tracking.

At the root of the problems of situation and threat assessment - of the deductive, abductive and inductive processes therein - are uncertainties in the quality of available data and available models of entities, environments, situations and of the measurement and exploitation process itself. These we may term epistemological and ontological uncertainties. Clearly, epistemological uncertainties derive, at least in a statistical sense, from ontological uncertainties: in our inability to accurately characterize the performance of available sensing, communications and data processing resources in sensing an unknown environment.

Therefore, as a background process (bottom right of Figure 2), the target, situation and sensor/source models are evaluated for their fidelity. It should be evident that this is also a data fusion problem, in which hypotheses diagnostic hypotheses - are generated to account for uncertainties in the target and situation assessment product. Such hypotheses can also be given the form of labeled directed graphs. These are often referred to as "fault trees"; although there is no reason to be restricted to singly-connected tree structures. Nor are we concerned only with "faults" in the sense of discrete losses of functionality; but with sources of random and deterministic errors throughout the estimation process.

Diagnostic hypotheses are evaluated and selected on the basis of their consistency with the evidence; i.e. by the extent to which uncertainties in the target and situation assessment product are explained by the ensemble of models.

\section{Estimation of Reporting Bias}

Let us distinguish a sensor's or information system's reporting bias - which may include intentional or unintentional human biases - from its measurement bias. The latter is but one contributor to the former.

We formulate the problem as that of simultaneously estimating states of multiple targets and of the values of systematic biases in the information sources that corrupt the reported observables. Constrained problems of this sort have been addressed in the area of multisensor/multi-target geolocation. We denote the entire collection of bias states across all of the sensors/sources by the vector $\beta$.

In the target tracking case, $\beta$ can be a multidimensional vector of position, ranging and azimuth errors for the entire set of sensing platforms in a surveillance region.

In the more general, "open-net" case, $\beta$ can be a multi-dimensional vector of bias terms to include measurement mis-calibration and biases in target state estimation or reporting by the sensor system (e.g. target classification, composition, location, activity, capability or intent estimation).

\subsection{Biases in Continuous Variables}

This is straight-forward in the case of continuous reporting elements: the sensor's reports of target position and kinematic state or of signal parameters. Numerous treatments of measurement biases are available (e.g. [7-13]). The assumption in all of these is that biases are globally due to uncompensated differences in

- sensor platforms' navigation state estimate (e.g. positional, heading or velocity uncertainty);

- sensors' position relative to the platform (including boresight and lever arm effects);

- sensors' calibration in its particular measurement space (e.g. measurement of pixel band intensity or of RF or of time epoch).

In all such cases, biases can be measured either (a) relatively - i.e. with respect to other sensors - or (b) absolutely - i.e. with respect to a universal standard, such as a standard spatiotemporal frame or measurement standard.

Following Keydel [7], biases in continuous variables can be represented as follows. Measurement $z_{s t}^{k}$ by sensor $S$ of a target $t$ in state $X_{t}^{k}$ at time $k$ is given by the expression

$$
z_{s t}^{k}=h_{s}^{k}\left(\beta, X_{t}^{k}\right)+v_{s t}^{k} ;
$$

where $h_{s}^{k}\left(\beta, X_{t}^{k}\right)$ is usually non-linear ${ }^{3}$ and $v_{s t}^{k}$ is zero-mean Gaussian noise with covariance matrix $R_{s}^{k}$. If the sensor is reporting honestly, $h_{s}^{k}\left(\beta, X_{t}^{k}\right)$ is a measurement function, incorporating all of the sensor's knowledge about the sensing geometry including platform location and pointing errors. In the more general case, we call $h_{s}^{k}\left(\beta, X_{t}^{k}\right)$ a reporting function,

\footnotetext{
${ }^{3}$ For a particular sensor $s, h_{s}^{k}$ will usually depend on only some of the bias states in $\beta$.
} 
which may include intentional or unintentional human bias as well.

Reporting biases in an individual sensor can be estimated either absolutely (i.e. with respect to a reference system) or relatively (i.e. with respect to other sensors).

In absolute registration, the preferred technique is to use fiducial targets - i.e. ones with known state - 'to observe the report bias directly.

In relative registration - generally performed in the absence of fiducial targets - one may use targets of opportunity to form a registration measurement set among $n$ sensors:

$$
Z=\left\{z_{s t}^{k} \mid s=1, \ldots, N_{s} ; t=1, \ldots, N_{t} ; k=1, \ldots, N_{k}\right\} \text {. }
$$

The registration set is a collection of measurements that nearly unambiguously correlate with their corresponding tracks.

The optimal estimate is obtained by noting that the dependence of the measurements is symmetric in the bias and target states. Therefore, simultaneous estimation of both the bias and target states can be performed, leading to a large Kalman filter problem. In practice, this is usually infeasible due to its large dimensionality.

To develop a tractable approximation to the optimal estimator, [7] approximates $z_{s t}^{k} \approx h_{s}^{k}\left(\beta, \hat{X}_{t}^{k \mid k}\right)+v_{s t}^{k}$; where $\hat{X}_{t}^{k \mid k}$ is the system level target state estimate, treated here as a parameter in the measurement equation.

$$
\text { A maximum likelihood bias estimate }
$$

$$
\hat{\beta}=\arg \max _{\beta} \Lambda(\beta)
$$

can then be obtained; e.g. using a Newton-Raphson or Levenberg-Marquardt search scheme. Here, $\Lambda(\beta)=-\frac{1}{2} \sum_{s, t, k}\left(z_{s t}^{k}-h_{s}^{k}\left(\beta, \hat{X}_{t}^{k \mid k}\right)\right)^{\mathrm{T}}\left(S_{s t}^{k}\right)^{-1}\left(z_{s t}^{k}-h_{s}^{k}\left(\beta, \hat{X}_{t}^{k \mid k}\right)\right)$ , and $S_{s t}^{k}$ is the innovation covariance; e.

A more innovative approach that allows for dynamic biases is to use an Extended Kalman filter for bias tracking [7]. The bias vector $\beta^{u}$ at time $u$ is then described by the Ito equation $d \beta^{u}=f\left(\beta^{u}\right) d t+g\left(\beta^{u}\right) d v^{u}$; where $f\left(\beta^{u}\right)$ and $g\left(\beta^{u}\right)$ will depend on the detailed nature of the sensor platforms. As each registration measurement becomes available, it is now processed using the Kalman filter equation

$$
\hat{\beta}^{k \mid k}=\hat{\beta}^{k \mid k-1}+W_{s t}^{k}\left(z_{s t}^{k}-h_{s}^{k}\left(\hat{\beta}^{k \mid k-1}, \hat{X}_{t}^{k \mid k}\right)\right) ;
$$

where $W_{s t}^{k}$ is the Kalman filter gain.

The measurement from sensor $s$ will generally couple to the bias states for all of the sensors in the constellation.

A classic method of solving such problems is that of Torrieri [8,9], in which the covariance matrix for each estimate is $P=\left[G^{T} N^{-1} G\right]^{-1}$; where the matrix of partials $G$ is computed at the converged solution.

$P$ can be partitioned so as to separate target state estimates and bias estimates:

$$
\begin{aligned}
P & =\left[\begin{array}{cc}
G_{11}^{T} & 0 \\
G_{12}^{T} & G_{22}^{T}
\end{array}\right]\left[\begin{array}{cc}
N_{11}^{-1} & 0 \\
G_{12}^{T} & N_{22}^{-1}
\end{array}\right]\left[\begin{array}{cc}
G_{11} & G_{12} \\
0 & G_{22}
\end{array}\right] \\
& =\left[\begin{array}{cc}
G_{11}^{T} N_{11}^{-1} G_{11} & G_{11}^{T} N_{11}^{-1} G_{12} \\
G_{12}^{T} N_{11}^{-1} G_{11} & G_{11}^{T} N_{11}^{-1} G_{12}+G_{22}^{T} N_{22}^{-1} G_{22}
\end{array}\right] .
\end{aligned}
$$

In contrast to such cases, we are concerned about patterns of biased reporting that may be due to intentional misrepresentation. A combatant may exaggerate the number of enemy combatants or the distance he traveled or his confidence in his assessment. A pirate may misrepresent the location of his buried treasure. The clear distinction is that such biases are not some simple continuous function across the measurement space; rather, such biases are discontinuous, reflecting specific patterns of distortion.

\subsection{Biases in Discrete Variables}

Cases of biases in reporting of discrete variables - in target type or activity state, for example - may result from unintentional errors in classification models. This is a common problem in military classification schemes, which rely on prior knowledge of the characteristics and behaviors of entities of interest and of other possible entities in the observation space that may be confused with the entities of interest. The effects of mis-modeling on classification performance are treated in [14].

Methods for measuring particular errors within a taxonomic scheme generally make use of a one or another semantic distance measure; or they incorporate empirical metrics [16-22].

A classification estimate can be represented as a vector from the true target class to some point in the $\mathrm{N}$ dimensional space defined by mutually orthogonal unit vectors corresponding to the $\mathrm{n}$ disjoint and independent classes. Thus every pair of target states uniformly has an implicit distance of $\sqrt{2}$.

\subsection{Patterned Biases}

Such simplistic models do not adequately represent the interesting real-world cases of reporting bias. For example, a toy manufacturer may be inclined to exaggerate the virtues of his products and of his business at the expense of those of his competitors. It does not follow that all of his reporting regarding all "targets" are likely to be biased. His political, religious and aesthetic biases are probably more or less independent of his biases concerning toys and toy companies. On the other hand, a manufacturer of military weapons may have subtle couplings between such biases.

Such considerations lead us to represent biases as functions of the structure of the source's evaluative space. This can incorporate epistemic and semantic models in ways that are not generally available for use as "calibration models".

When reporting or response involves a human element - whether as a system user or as a system designer - the problem of characterizing, predicting and recognizing reporting or other response biases is seen to 
be a problem of the sort that we are pursuing via the apparatus described in Section 3: a problem of characterizing, predicting and recognizing intentional activity.

Consideration of an agent's repertoire of actions and his expectations as to outcome and subjective utility will be used to characterize discrepancies in reporting. These can be evaluated either absolutely - as variance from assumed truth - or relative to other sources.

\subsection{Passive and Active Information Acquisition}

The lack of indicators of reporting biases in available information sources is a serious problem in data fusion, particularly when, as in the Network Centric paradigm, information networks are envisioned as unconstrained, ad hoc assemblages. Given the likelihood of private agendas, not to mention intentional malicious distortions of information, we seek methods to predict and recognize such biases.

One potentially useful method that we are exploring is that of Stimulative Intelligence; meaning the systematic stimulation of agents or their environment to elicit information [23]. Such stimulation can be physical (e.g. imparting energy to stimulate a kinetic, thermal, or reflective response), informational (e.g. providing false or misleading information), or psychological (e.g. stimulating perceptions, emotions or intentions). This results in a revision of the architecture shown in Figure 2 to that of Figure 4.

The augmented threat assessment process closes the loop by means of a response management function (at the left of the figure) that nominates information collection actions that - with some estimated probabilities - can support the current decision needs. These actions can include the intentional stimulation of the information environment - including information sources of all stripes - to elicit information.

Stimulation that can be expected to induce information sources to reveal their biases requires an inventory of models for various classes of behavior. The hope is that methods for the systematic discovery and generalization of such models - the abductive and inductive elements of Figure 4 - can be developed.

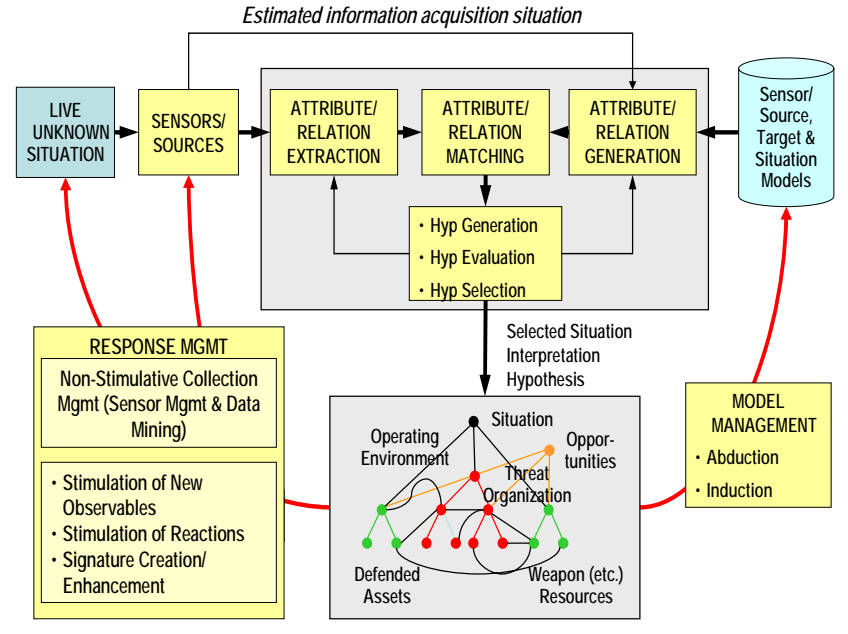

Figure 4. Integrating Stimulative Intelligence into the Information Acquisition Process

\section{References}

[1] Tom Spring, “Next generation spam”, PC World, January 24, 2006.

[2] H.P. Grice, "Logic and conversation," The Logic of Grammar, ed. Donald Davidson and Gilbert Harman (Encino, Cal.: Dickenson), pp. 64-75, 1975.

[3] Alberts and Hayes, "Power to the Edge", http://www.dodccrp.org/publications/pdf/Alberts_ Power.pdf .

[4] Alan N. Steinberg, "Unification across data fusion levels 1-3”, Proceedings, Seventh International Conference on Information Fusion, Stockhom, 2004.

[5] Alan N. Steinberg, "An approach to threat assessment”, Proceedings, Eighth International Conference on Information Fusion, Philadelphia, D8-4, 2005.

[6] Eric Little, “Ontology meta-modeling for building a Situational picture of catastrophic events", Proceedings, Eighth International Conference on Information Fusion, Philadelphia, 2005.

[7] Eric R. Keydel, “Multi-INT registration issues”, IDGA Image Fusion Conference, Washington, 1999.

[8] Don J, Torrieri, "Statistical theory of passive location systems", IEEE Transactions on Aerospace and Electronic Systems, Vol. AES-20, No. 2. March, 1984.

[9] C. Robert Mathewson, "MSEML - a data-driven solution for SIGINT geolocation”, (conference paper), 2001.

[10] Lin, X., Kirubarajan, T., and Bar-Shalom, Y., "Multisensor bias estimation with local tracks without a priori association", Proceedings of SPIE Conference on Signal and Data Processing of 
Small Targets, Vol. 5204, San Diego, CA, August 2003.

[11] Lin, X., Kirubarajan, T., and Bar-Shalom, Y., "Exact multisensor dynamic bias estimation with local tracks", Proc. Fusion 2003, The 6th International Conference on Information Fusion, Queensland, Australia, July 2003.

[12] Lin, X., Kirubarajan, T., and Bar-Shalom, Y., "Multisensor-Multitarget Bias Estimation for Asynchronous Sensors". Proceedings of SPIE Conference on Signal Processing, Sensor Fusion, and Target Recognition XIII, Vol. 5429, Orlando, FL, April 2004.

[13] Lin, X., Kirubarajan, T., and Bar-Shalom, Y., "Multisensor-multitarget bias estimation for general asynchronous sensors”, Proc. Fusion 2004, The 7th International Conference on Information Fusion, Stockholm, July 2004.

[14] Lawrence Stone and Thuy Tran, "Effect of model uncertainty on target discrimination", Workshop on Estimation, Tracking and Fusion: A Tribute to Yaakov Bar-Shalom, Monterrey, CA, 2001.

[15] A.D. Gordon, Classification, Second Edition, Chapman \& Hall/CEC, Washington, DC, 1999.

[16] Alexander Budanitsky, Lexical Semantic Relatedness and its Application in Natural Language Processing, technical report CSRG-390, Department of Computer Science, University of Toronto, August 1999.

[17] Alexander Budanitsky and Graeme Hirst, "Semantic distance in WordNet: an experimental application-oriented evaluation of five measures", http://www.cs.toronto.edu/compling/Publications/ Abstracts/Papers/Budanitsky+Hirst-2001-abs.html.

[18] Graeme Hirst and David St-Onge. 1998. "Lexical chains as representations of context for the detection and correction of malapropisms", WordNet: An Electronic Lexical Database, ed. Christiane Fellbaum, The MIT Press. Fellbaum, pp. 305-332, 1998.

[19] Philip Resnik, "Using information content to evaluate semantic similarity. Proceedings of the 14th International Joint Conference on Artificial Intelligence, pages 448-453, Montreal, 1995.

[20] Dekang Lin. 1998. An information-theoretic definition of similarity”, Proceedings of the 15th International Conference on Machine Learning, Madison, WI, 1998.

[21] Claudia Leacock and Martin Chodorow, "Combining local context and WordNet similarity for word sense identification”, WordNet: An Electronic Lexical Database, ed. Christiane Fellbaum, The MIT Press. Fellbaum, pp. 265-283, 1998.
[22] Jay J. Jiang and David W. Conrath, "Semantic similarity based on corpus statistics and lexical taxonomy", Proceedings of International Conference on Research in Computational Linguistics, Taiwan, 1997.

Alan N. Steinberg, "Stimulative intelligence”, Proceedings of the National Symposium on Sensor and Data Fusion, 2006. 